\title{
Optimal selection of tests for fault detection and isolation in multi-operating mode system
}

\author{
LIU Yuanhong* \\ School of Equipment Engineering, Armed Police Force Engineering University, Xi' an 710086, China
}

\begin{abstract}
The complex systems are often in the structure of multi-operating modes, and the components implementing system functions are different under different operation modes, which results in the problems that components often fail in different operating modes, faults can be only detected in specified operating modes, tests can be available in specified operating modes, and the cost and efficiency of detecting and isolating faults are different under different operating modes and isolation levels. Aiming at these problems, an optimal test selection method for fault detection and isolation in the multi-operating mode system is proposed by using the fault pair coding and rollout algorithm. Firstly, the faults in fault-test correlation matrices under different operating modes are combined to fault-pairs, which is used to construct the fault pair-test correlation matrices under different operating modes. Secondly, the final fault pair-test correlation matrix of the multioperating mode system is obtained by operating the fault pair-test correlation matrices under different operating modes. Based on the final fault pair-test correlation matrix, the necessary tests are selected by the rollout algorithm orderly. Finally, the effectiveness of the proposed method is verified by examples of the optimal test selection in the multi-operating mode system with faults isolated to different levels. The result shows that the proposed method can effectively mine the fault detection and isolation ability of tests and it is suitable for the optimal test selection of the multi-operating mode system with faults isolated to the replacement unit and specific fault.
\end{abstract}

Keywords: operating mode, test optimization, fault pair coding, fault detection, fault isolation.

DOI: $10.21629 / J S E E .2019 .02 .20$

\section{Introduction}

With the extensive application of the testability technology in the complex equipment such as spacecraft, aircraft, ship and armored vehicles, the testability design method research in the complex equipment becomes one of the

\footnotetext{
Manuscript received December 07, 2017.

*Corresponding author.

This work was supported by the Natural Science Foundation of Shannxi Province (2017JQ5016), and the Joint Laboratory for Sea Measurement and Control of Aircraft (DOM2016OF011).
}

hotspots in the field of testability [1]. The practical equipment is mostly multi-level in the functional structure, a plurality of components constitute a relatively independent replaceable unit, and a plurality of replaceable units constitute the subsystem and system [2-8]. There are a variety of operating modes in the practical equipment, and the replaceable units and subsystems that make up the system often have a variety of operation modes, which results in a variety of faults in the system, faults under different parts, replaceable units and subsystems are also different and can be measured only in the specified operation mode, most of the tests work in the specified operation mode, and the cost and efficiency of detecting and isolating faults at different isolation levels in the same operating mode are different. Therefore, to achieve accurate, fast and comprehensive testing and diagnosis of the multi-operating mode system at the lowest cost, key techniques of testability modeling, optimal test selection, and diagnostic strategy optimization for the multi-operating mode system need to be studied.

The purpose of the optimal test selection is to find the best test set to meet testability requirements in all possible test sets. The testability engineering and maintenance system (TEAMS) software proposed by qualtech systems incorporation (QSI) [9-12] and the eXpress software proposed by diagnostic system incorporation (DSI) [13] can generate the optimal diagnostic strategy based on the optimal test set. However, the test optimization process is a round-robin process, that is, the testability indicators are calculated based on the fault-test correlation matrices under different operating modes to determine whether to meet some requirements and whether to add tests, the software cannot automatically generate an optimal test set. Mathematically speaking, the optimal test selection problem of the multi-operating mode system is a combinatorial optimization problem. The existing optimal test selection methods such as various evolutionary algorithms (such as the genetic algorithm [14-17], simulated annealing algorithm [18], particle swarm optimiza- 
tion algorithm [19-21], quantum-inspired evolutionary algorithm [22], firefly algorithm [23-24]), greedy algorithm [25], Boolean logic algorithm [26] have achieved good results under a single operation mode. But the existing algorithms are directly fitted for the single fault-test correlation matrix. There are a variety of operating modes in some systems. Different components from the system under different modes, which results in different faults under different operating modes. That is, when the optimal test selection is applied to the multi-operating mode system, there are several different fault-test correlation matrices, and different levels of systems tend to be different levels of isolation; therefore, a new optimal test selection method is proposed by using the fault pair coding and rollout heuristic search algorithm.

\section{Optimal test selection of multi-operating mode system}

Assuming that the fault-test correlation matrix of a multioperating mode system is shown in Table 1 , and all tests are symmetrical. The system consists of $R$ replaceable units, including $N$ operating modes and $m+1$ statuses $\left\{f_{0}, f_{1}, f_{2}, \ldots, f_{m}\right\}$. $f_{0}$ denotes a normal status. $\left\{f_{1}, f_{2}, \ldots, f_{m}\right\}$ denotes $m$ kinds of fault statuses, and the corresponding probability set is $\left\{p_{0}, p_{1}, p_{2}, \ldots, p_{m}\right\}$. $\left\{T_{1}, T_{2}, \ldots, T_{K}\right\}$ denotes the test set including $K$ tests, and the corresponding test cost set is $\left\{C_{1}, C_{2}, \ldots, C_{K}\right\}$, the test $T_{k}$ under the $r$ th operating mode is expressed as $\left\{t_{r 1}, t_{r 2}, \ldots, t_{r K}\right\}$. The value of $d_{i j}^{r}$ is 0 or 1 , which denotes the correlation between the test $t_{r j}$ and the fault $f_{i}$ (related or uncorrelated) under the $r$ th operating mode.

Table 1 Fault-test dependency matrix of multi-operating mode system

\begin{tabular}{|c|c|c|c|c|c|c|c|c|c|c|c|c|c|c|}
\hline \multirow{2}{*}{ Fault } & \multicolumn{4}{|c|}{ Operating mode 1} & \multicolumn{4}{|c|}{ Operating mode 2} & & \multicolumn{5}{|c|}{ Operating mode $N$} \\
\hline & $t_{11}$ & $t_{12}$ & $\cdots$ & $t_{1 K}$ & $t_{21}$ & $t_{22}$ & $\cdots$ & $t_{2 K}$ & $\cdots$ & $t_{N 1}$ & $t_{N 2}$ & $\cdots$ & $t_{N K}$ & \\
\hline$f_{0}$ & 0 & 0 & $\cdots$ & 0 & 0 & 0 & $\cdots$ & 0 & $\cdots$ & 0 & 0 & $\cdots$ & 0 & $p_{0}$ \\
\hline$f_{1}$ & $d_{11}^{1}$ & $d_{12}^{1}$ & $\cdots$ & $d_{1 K}^{1}$ & $d_{11}^{2}$ & $d_{12}^{2}$ & $\cdots$ & $d_{1 K}^{2}$ & $\cdots$ & $d_{11}^{N}$ & $d_{12}^{N}$ & $\cdots$ & $d_{1 K}^{N}$ & $p_{1}$ \\
\hline : & $\vdots$ & $\vdots$ & $\ddots$ & $\vdots$ & $\vdots$ & $\vdots$ & $\ddots$ & $\vdots$ & $\ddots$ & $\vdots$ & $\vdots$ & $\ddots$ & $\vdots$ & $\vdots$ \\
\hline$f_{m}$ & $d_{m 1}^{1}$ & $d_{m 2}^{1}$ & $\cdots$ & $d_{m K}^{1}$ & $d_{m 1}^{2}$ & $d_{m 2}^{2}$ & $\cdots$ & $d_{m K}^{2}$ & $\cdots$ & $d_{m 1}^{N}$ & $d_{m 2}^{N}$ & $\cdots$ & $d_{m K}^{N}$ & $p_{m}$ \\
\hline
\end{tabular}

When the test is of multi-value, the value of $d_{i j}^{r}$ is of multi-value, indicating the corresponding output values of the test $t_{r j}$ when the fault $f_{i}$ occurs under the $r$ th operating mode.

In the optimal test selection of the multi-operating mode system, the optimal test set is found in fault-test correlation matrices of the determined operating modes to obtain the optimal fault detection rate and the fault isolation rate at the lowest cost.

The optimal test selection process mainly includes fault detection and fault isolation [16]. In an operating mode, the sufficient and necessary condition that a fault can be detected is that when the fault occurs, there is a test at least, whose output result is abnormal, that is, not all for " 0 " in the fault-test correlation matrix.

The necessary and sufficient condition for a fault to be isolated is that when the fault occurs, the test result is different from the outputs of other faults, that is, the row vector in the fault-test correlation matrix is different from other line vectors. Based on this idea, the fault pair-test correlation matrix of the multi-operating mode system is obtained by fault pair coding based on the fault-test correlation matrices of different operating modes, and the fault detection and isolation process is transformed into seeking the optimal test set process for detecting fault pairs based on the fault pair-test correlation matrix. The fault pairs include fault and normal pairs, fault and fault pairs, and the normal state is expressed as a special fault for the convenience of narration.

\section{Fault pair coding}

Assuming $D_{n}\left(f_{i}\right)$ denotes the test set for detecting the fault $f_{i}$ under the $n$th operating mode. The sufficient and necessary condition for the test $t_{n k}$ detecting the fault $f_{i}$ is $d_{i k}^{n}=1$. Thus, the test set that can detect the fault $f_{i}$ is expressed as follows:

$$
D_{n}\left(f_{i}\right)=\sum_{k=1}^{K}\left[d_{i k}^{n}=1\right] .
$$

The necessary and sufficient condition for test $t_{n j}$ distinguishing the fault $f_{i}$ and the fault $f_{j}$ is $d_{i k}^{n} \oplus d^{n}+j k=1$, and $\oplus$ is the exclusive and/or operation. Thus, the test set $I\left(f_{i}, f_{j}\right)$ for distinguishing the fault $f_{i}$ and the fault $f_{j}$ is expressed as follows:

$$
I\left(f_{i}, f_{j}\right)=\sum_{k=1}^{K}\left(d_{i k}^{n} \oplus d_{j k}^{n}=1\right) .
$$

The test set $I\left(f_{0}, f_{i}\right)$ for distinguishing the fault $f_{0}$ and the fault $f_{i}$ is expressed as follows:

$$
I\left(f_{0}, f_{i}\right)=\sum_{k=1}^{K}\left(0 \oplus d_{i k}^{n}=1\right)=\sum_{k=1}^{K}\left(d_{i k}^{n}=1\right) .
$$

From (1) and (3), it can be seen that in each operating mode, the test set that can detect the fault $f_{i}$ is equal to 
the test set that can distinguish the fault $f_{0}$ and $f_{i}$. Therefore, the search for the smallest test set to isolate the faults under a certain operating mode is transformed to seek the optimal test set for distinguishing all the fault pairs composed of system faults. The test can distinguish the fault pair according to (2).

The fault detection rate indicates the ratio of the number of faults that can be detected to the total number of the system faults, which has nothing to do with the maintenance level. The fault isolation rate is a ratio of the number of faults that can be isolated to the specified level to the total number of faults that can be detected [27]. When the faults need to be isolated to the replaceable unit, the faults of different replaceable units are actually separated from each other, while the faults of the same unit do not need to be distinguished. Thus, it is assumed that $U=\left\{U_{0}, U_{1}, \ldots, U_{l}\right\}$ denotes the replaceable unit set. $U_{0}$ denotes that the system is normal, and isolating the faults to the replaceable units is practically differentiated from all the fault pairs composed by the faults in different replaceable units without the need to distinguish the faults pairs composed by the faults in the same replaceable unit.

The necessary and sufficient conditions for $T_{k}$ detecting the fault $f_{i}$ under the multi-operating mode system is expressed as follows:

$$
\left(d_{i k}^{1}\left\|d_{i k}^{2} \cdots\right\| d_{i k}^{N}\right)=1 .
$$

In (4), " "|" denotes the OR computation. Therefore, the test set for detecting the fault $f_{i}$ is expressed as follows:

$$
D\left(f_{i}\right)=\sum_{k=1}^{K}\left[\left(d_{i k}^{1}\left\|d_{i k}^{2} \cdots\right\| d_{i k}^{N}\right)=1\right]
$$

The necessary and sufficient conditions for $T_{k}$ distinguishing the faults $f_{i}$ and $f_{j}$ under the multi-operating mode system is expressed as follows:

$$
\left(d_{i k}^{1} \oplus d_{j k}^{1}\right)\left\|\left(d_{i k}^{2} \oplus d_{j k}^{2}\right)\right\| \cdots \|\left(d_{i k}^{N} \oplus d_{j k}^{N}\right)=1 .
$$

The test set $I\left(f_{i} f_{j}\right)$ for distinguishing the fault $f_{i}$ and $f_{j}$ in the multi-operating mode system is expressed as follows:

$$
\begin{gathered}
I\left(f_{i}, f_{j}\right)=\sum_{k=1}^{K}\left\{\left(d_{i k}^{1} \oplus d_{j k}^{1}\right)\left\|\left(d_{i k}^{2} \oplus d_{j k}^{2}\right)\right\| \cdots\right. \\
\left.\|\left(d_{i k}^{N} \oplus d_{j k}^{N}\right)=1\right\} .
\end{gathered}
$$

The test set $I\left(f_{0}, f_{i}\right)$ for distinguishing the fault $f_{0}$ and $f_{i}$ in the multi-operating mode system is expressed as follows:

$$
I\left(f_{0}, f_{j}\right)=\sum_{k=1}^{K}\left\{\left(0 \oplus d_{j k}^{1}\right)\left\|\left(0 \oplus d_{j k}^{2}\right)\right\| \cdots \|\left(0 \oplus d_{j k}^{N}\right)=1\right\}=
$$

$$
\sum_{k=1}^{K}\left[\left(d_{i k}^{1}\left\|d_{i k}^{2} \cdots\right\| d_{i k}^{N}\right)=1\right]=D\left(f_{i}\right) .
$$

From (6) and (8), it can be seen that the test set capable of detecting faults in the multi-operating mode system is equal to the test set that can distinguish the fault pair $f_{0}$ and $f_{i}$. From (7) and (8), it can be found that seeking the minimum test set for the multi-operating mode system is equal to seeking the minimum test set for distinguishing all the possible fault pairs.

Therefore, for the multi-operating mode system, a fault pair coding method is proposed. Firstly, the test distinguishing the faults under the $r$ th operating mode can be judged according to (2) and (8). Then, the fault pair-test matrix $\left[d p_{i j}^{r l}\right]$ is built, in which the columns represent fault pairs, and the rows represent tests. $d p_{i j}^{r l}=1$ denotes that the test $t_{r l}$ can distinguish the faults $f_{i}$ and $f_{j}$, otherwise, $d p_{i j}^{r l}=0$. Finally, whether or not the test $T_{l}$ can distinguish fault pairs according to (6) is determined. That is, the final fault pair-test correlation matrix $\left[D P_{i j}^{l}\right]$ is obtained by the OR computation of each fault pair-test matrix under each operating mode, which is shown as follows:

$$
D P_{i j}^{l}=\left(d p_{i j}^{1 l}\left\|d p_{i j}^{2 l}\right\| d p_{i j}^{3 l}\|\cdots\| d p_{i j}^{N l}\right) .
$$

\section{Rollout heuristic search algorithm}

The rollout algorithm is proposed by Bertskas et al. [28], which is originally used to solve the combinatorial optimization and random ordering problems. The algorithm consists of a benchmark search strategy and an iterative strategy. The main idea is to use the iterative algorithm to get the update strategy, and then use the update strategy as a reference strategy to update and gradually get the optimal strategy $[29,30]$. The rollout heuristic search algorithm is used to optimize the test selection of the multi-operating mode system. Its core lies in the construction of the benchmark heuristic search strategy and the heuristic iterative update strategy.

\subsection{Benchmark heuristic search strategy}

The benchmark heuristic search strategy uses the greedy search, and the key of the greedy search is to build the heuristic function. In a fault pair-test matrix, the fault pair can be distinguished by more tests, the higher the fault probability is, the easier it is to detect and isolate the fault. Meanwhile, the lower the selected test costs, the better the test is. Therefore, the heuristic function of the benchmark heuristic search strategy is put forward as follows by integrating the test cost, test fault detection and isolation ability.

$$
k^{*}=\arg \max _{j}\left\{A\left(F P, t_{j}\right) / c_{j}\right\}
$$


where $c_{j}$ is test cost, $A\left(F P, t_{j}\right)$ is the fault pair detection ability of $t_{j}$ with the fault pair set $F P$, which is calculated as follows:

$$
A\left(F P, t_{j}\right)=\sum_{i=1}^{N_{P}} D_{i}\left(F_{s}, t_{j}\right) P_{i}\left(F_{s}, t_{j}\right)
$$

where $F_{s}$ is the fault pair set that can be distinguished by the test $t_{j}$ under $F P$. That is, the column value of $D P_{i j}^{l}$ that $t_{j}$ corresponds in the fault pair-test matrix $\left[D P_{i j}^{l}\right]$ is 1 . $N_{P}$ is the number of fault pairs, $D_{i}\left(F_{s}, t_{j}\right)$ is the probability that the $i$ th fault pair in $F_{s}$ can be distinguished by the test set, $P_{i}\left(F_{s}, t_{j}\right)$ is the fault pair probability by the multiplication of the two faults.

Assume that the fault pair-test correlation matrix is $F P-T$, the number of the test set $T$ is $N_{T}$, the $i$ th fault pair can be distinguished by $k_{i}$ tests, then the $D_{i}\left(F_{s}, t_{j}\right)$ can be calculated as follows:

$$
D_{i}\left(F_{s}, t_{j}\right)=\frac{k_{i}}{N_{T}} .
$$

The benchmark strategy is to select the maximum value test $t_{\max }$ according to (10) from the available test set in a test selection process until all the fault pairs are differentiated to meet the fault detection and isolation requirements. The specific steps are as follows.

Step 1 Initialize the optimal test set $T_{\text {Flopt }}$ as empty, and the remaining fault pair-test correlation matrix is $\left[D P_{i j}^{l}\right]$.

Step 2 According to (10) and the residual fault pair-test correlation matrix, calculate the corresponding value of $k$ in (10), select the largest value corresponding test $t_{\max }$, that is, the best test. Add the $t_{\max }$ to $T_{\text {Flopt }}$, meanwhile the rows in the remaining fault pair-test that the test $t_{\max }$ can detect are removed. That is, delete the rows that $t_{\max }$ corresponds, in which the output value of the test $t_{\max }$ is 1 , then delete the column that $t_{\max }$ corresponds, and get the new residual fault pair-test correlation matrix.

Step 3 If the new remaining fault pair-test correlation matrix is empty, that is, all the fault pairs can be detected by the remaining tests, or no fault pair can be detected, then the algorithm exits; otherwise turn to Step 2.

\subsection{Heuristic irrelative updating strategy}

Step 1 Assume that the fault pair set to be distinguished is $F P$, the test set is $T$, then select the test $t_{i}$ in $T$, delete the rows in $\left[D P_{i j}^{l}\right]$ that $t_{i}$ can detect, and delete $t_{i}$ to get the remaining fault pair-test correlation matrix.

Step 2 Use the benchmark heuristic search strategy to obtain the local optimal test set with the residual fault pairtest correlation matrix. The lower the test costs, the better the test set is, the better the fault detection rate and the fault isolation rate are. Therefore, the evaluation function of the test set by the benchmark heuristic search strategy is proposed as follows:

$$
J_{i}=\left(\sum_{j=1}^{N} c_{j}+c_{i}\right) /\left(R_{\mathrm{FDR}} \cdot R_{\mathrm{FIR}}\right)
$$

where the parameters $R_{\mathrm{FDR}}$ and $R_{\mathrm{FDR}}$ denote the fault detection rate and the fault isolation rate respectively, which are calculated according to the standard MIL-STD-2165A [31]. Select the minimum value of the evaluation function $J_{i}$. When the test sets under $t_{i}$ and $t_{j}$ are the same, the evaluation function is equal. Therefore, according to (10), select the optimal test between $t_{i}$ and $t_{j}$, and realize the excellent selection of excellent tests.

Step 3 In the fault pair-test correlation matrix, delete the fault pair rows that the test $t_{\max }$ can detect. That is, the rows in the fault pair-test correlation matrix that the output value of $t_{\max }$ is equal to 1 . Then delete the test $t_{\max }$ to get the new fault pair-test correlation matrix.

Step 4 If the remaining fault pair-test correlation matrix is empty. That is, all fault pairs can be detected, or no fault can be distinguished. The algorithm exits; otherwise turns to Step 2.

\section{Numerical case analysis}

\subsection{Optimal test selection with isolation to fault}

The fault-test correlation matrix for the multi-operating mode system is shown in Table 2.

In Table 2, the system consists of seven replaceable units (the replacement unit $U_{0}$ denotes the system normal state), there are two kinds of operating modes, and seven replaceable units have 11 faults. The test cost is shown in Table 3.

With isolation to faults, the fault pair-test matrices of the two operating modes is obtained by coding the fault-test correlation matrices of operating mode 1 and mode 2 in Table 2 according to (6) and (8). Then, the two fault pair-test correlation matrices are calculated by the OR computation, and the final fault pair-test matrix is obtained as shown in Table 4. The number of fault pairs is $C_{11}^{2}=55$ in Table 4. Assuming the faults are independent of each other, the probabilities of fault pairs are the products of the two faults.

According to Table 4, the optimal test selection process by using the rollout heuristic search algorithm is shown in Fig. 1.

Firstly, the optimal test set is obtained by using the benchmark heuristic search strategy. For example, if the test $T_{1}$ is selected, delete the rows in the fault pair-test cor- 
relation matrix in Table 3 that the test $T_{1}$ can detect, and then delete the column in Table 3 that the test $T_{1}$ lies in and get the remaining fault pair-test correlation matrix and the remaining test set. In this way, the optimal test sequence
$\left\{T_{7}, T_{4}, T_{6}, T_{5}\right\}$ under the test $T_{1}$ is obtained by using the benchmark heuristic search strategy. That is, the local optimal test set is $\left\{\begin{array}{lllll}T_{1} & T_{7} & T_{4} & T_{6} & T_{5}\end{array}\right\}$, when the test $T_{1}$ is selected.

Table 2 Fault-test correlation matrix for multi-operating mode system

\begin{tabular}{|c|c|c|c|c|c|c|c|c|c|c|c|c|c|c|c|c|}
\hline \multirow{2}{*}{ Fault } & \multirow{2}{*}{$\begin{array}{c}\text { Subject } \\
\text { unit }\end{array}$} & \multicolumn{7}{|c|}{ Operating mode 1} & \multicolumn{7}{|c|}{ Operating mode 2} & \multirow{2}{*}{ Probability } \\
\hline & & $t_{11}$ & $t_{12}$ & $t_{13}$ & $t_{14}$ & $t_{15}$ & $t_{16}$ & $t_{17}$ & $t_{21}$ & $t_{22}$ & $t_{23}$ & $t_{24}$ & $t_{25}$ & $t_{26}$ & $t_{27}$ & \\
\hline$f_{0}$ & $\overline{U_{0}}$ & 0 & 0 & 0 & 0 & 0 & 0 & 0 & 0 & 0 & 0 & 0 & 0 & 0 & 0 & 0.7000 \\
\hline$f_{1}$ & $U_{1}$ & 0 & 0 & 0 & 0 & 0 & 1 & 0 & 0 & 0 & 0 & 0 & 0 & 0 & 0 & 0.0302 \\
\hline$f_{2}$ & $U_{2}$ & 0 & 1 & 0 & 1 & 0 & 0 & 0 & 0 & 0 & 0 & 0 & 0 & 0 & 0 & 0.0505 \\
\hline$f_{3}$ & $U_{2}$ & 0 & 0 & 0 & 0 & 1 & 0 & 0 & 0 & 0 & 0 & 0 & 0 & 0 & 0 & 0.0132 \\
\hline$f_{4}$ & $U_{2}$ & 1 & 1 & 1 & 1 & 0 & 1 & 0 & 1 & 1 & 0 & 0 & 0 & 0 & 0 & 0.0544 \\
\hline$f_{5}$ & $U_{3}$ & 1 & 1 & 1 & 1 & 1 & 0 & 0 & 0 & 1 & 1 & 0 & 0 & 0 & 0 & 0.0024 \\
\hline$f_{6}$ & $U_{3}$ & 0 & 0 & 0 & 0 & 1 & 0 & 1 & 0 & 0 & 0 & 0 & 0 & 0 & 0 & 0.0213 \\
\hline$f_{7}$ & $U_{3}$ & 0 & 0 & 0 & 0 & 1 & 1 & 1 & 0 & 0 & 0 & 0 & 0 & 0 & 0 & 0.0036 \\
\hline$f_{8}$ & $U_{4}$ & 0 & 0 & 0 & 0 & 0 & 1 & 1 & 0 & 0 & 0 & 0 & 0 & 0 & 0 & 0.0075 \\
\hline$f_{9}$ & $U_{4}$ & 0 & 0 & 0 & 0 & 1 & 0 & 1 & 0 & 0 & 1 & 1 & 0 & 0 & 0 & 0.0634 \\
\hline$f_{10}$ & $U_{4}$ & 0 & 0 & 0 & 0 & 0 & 1 & 1 & 1 & 0 & 0 & 1 & 0 & 0 & 0 & 0.0535 \\
\hline
\end{tabular}

Table 3 Test cost

\begin{tabular}{|c|c|c|c|c|c|c|c|c|c|c|}
\hline \multirow{2}{*}{$\begin{array}{c}\text { Test name } \\
\text { Test cost }\end{array}$} & $T_{1}$ & \multicolumn{2}{|c|}{$\overline{T_{2}}$} & $T_{3}$ & \multicolumn{2}{|c|}{$T_{4}$} & \multicolumn{2}{|l|}{$T_{5}$} & \multirow{2}{*}{$\frac{T_{6}}{1}$} & \multirow{2}{*}{$\begin{array}{c}T_{7} \\
0.5\end{array}$} \\
\hline & 1 & & 1 & 1 & & 1 & 1 & & & \\
\hline \multicolumn{2}{|r|}{ Table 4} & \multicolumn{9}{|c|}{ Fault pair-test correlation matrix } \\
\hline $\begin{array}{c}\text { Serial } \\
\text { number }\end{array}$ & $\begin{array}{l}\text { Fault } \\
\text { pair }\end{array}$ & $T_{1}$ & $T_{2}$ & $T_{3}$ & $T_{4}$ & $T_{5}$ & $T_{6}$ & $T_{7}$ & Pro & ability \\
\hline 1 & $\left(f_{0} f_{1}\right)$ & 0 & 0 & 0 & 0 & 0 & 1 & 0 & & 2114 \\
\hline 2 & $\left(f_{0} f_{2}\right)$ & 0 & 1 & 0 & 1 & 0 & 0 & 0 & & 3535 \\
\hline$\vdots$ & $\vdots$ & $\vdots$ & $\vdots$ & $\vdots$ & $\vdots$ & $\vdots$ & $\vdots$ & $\vdots$ & & $\vdots$ \\
\hline 45 & $\left(f_{5} f_{10}\right)$ & 1 & 1 & 1 & 1 & 1 & 1 & 1 & & 0013 \\
\hline 46 & $\left(f_{6} f_{7}\right)$ & 0 & 0 & 0 & 0 & 0 & 1 & 0 & & 0008 \\
\hline 47 & $\left(f_{6} f_{8}\right)$ & 0 & 0 & 0 & 0 & 1 & 1 & 0 & & 0016 \\
\hline : & : & : & : & : & : & : & : & : & & : \\
\hline 49 & $\left(f_{6} f_{10}\right)$ & 1 & 0 & 0 & 1 & 1 & 1 & 0 & & 0114 \\
\hline 50 & $\left(f_{7} f_{8}\right)$ & 0 & 0 & 0 & 0 & 1 & 0 & 0 & & 0003 \\
\hline 51 & $\left(f_{7} f_{9}\right)$ & 0 & 0 & 1 & 1 & 0 & 1 & 0 & & 0023 \\
\hline : & : & : & . & : & : & : & : & : & & : \\
\hline 55 & $\left(f_{9} f_{10}\right)$ & 1 & 0 & 1 & 0 & 1 & 1 & 0 & & 0339 \\
\hline
\end{tabular}

The optimal test sets for the test $T_{2}, T_{3}, T_{4}, T_{5}, T_{6}$ and $T_{7}$ are $\left\{T_{2} T_{7} T_{6} T_{5} T_{4}\right\},\left\{T_{3} T_{4} T_{6} T_{7} T_{5}\right\},\left\{T_{4} T_{6} T_{7} T_{5}\right\}$, $\left\{T_{5} T_{4} T_{6} T_{7}\right\},\left\{T_{6} T_{7} T_{4} T_{5}\right\}$ and $\left\{T_{7} T_{2} T_{6} T_{5} T_{4}\right\}$, respectively. As all the faults are detected and isolated, the fault detection rate and isolation rate are all $100 \%$, and the evaluation function values are equal to the test cost according to (13), which are $\{4.5,4.5,4.5,3.5,3.5,3.5,4.5\}$. The corresponding test sets are $\left\{T_{4} T_{6} T_{7} T_{5}\right\},\left\{T_{5} T_{4} T_{6} T_{7}\right\}$, $\left\{T_{6} T_{7} T_{4} T_{5}\right\}$ respectively, when the evaluation function value is the minimum. That is, select one of the test sets $\left\{T_{4} T_{5} T_{6}\right\}$. As its evaluation function values are equal, the heuristic function values according to (10) are $\{0.3719$,
$0.2344,0.3274\}$. Therefore, the test $T_{4}$ is finally selected in candidates $\left\{T_{4} T_{5} T_{6}\right\}$. The other remain optimal tests are selected in the same way, and the final optimal test set is $\left\{T_{4} T_{7} T_{5} T_{6}\right\}$. The residual fault pair-test correlation matrix is empty, that is, all the faults are detected and isolated.

According to (10), the test $T_{1}$ is selected first by using the benchmark heuristic search strategy, and the final optimal test set is $\left\{T_{1} T_{7} T_{4} T_{6} T_{5}\right\}$ as shown in Fig.1. The test number and the test cost are higher than those obtained by the proposed rollout heuristic search algorithm.

In order to verify the effectiveness of the proposed algorithm, a typical evolutionary algorithm-genetic algorithm is constructed for the optimal test selection. In the calculation process, a fitness function is constructed as $f(T)=$ $\left[R(T)_{D} / R_{D}\right]^{2} \cdot\left[R(T)_{I} / R_{I}\right]^{2} \cdot\left[1-C(T) / C_{Z}\right]$ by integrating the fault detection rate, the fault isolation rate and the test cost, $R(T)_{D}$ and $R(T)_{I}$ are respectively the fault detection rate and the fault isolation rate of the selected test set $T$, which are set to $100 \%$ respectively. $C(T)$ is the test cost of the selected test set $T . C_{Z}$ is the total cost of all tests in the fault-test correlation matrix. The initial population size is set to 20 , the maximum number of iterations is set to 200 , and the mutation probability is set to 0.1 , the crossover probability is set to 0.8 . The final optimal test set is $\left\{T_{4} T_{5} T_{6} T_{7}\right\}$ by the initialized genetic algorithm, the fault detection rate and the fault isolation rate are both $100 \%$, and the maximum fitness function value is $1-3.5 / 6.5=0.4615$. The optimal solution obtained by the proposed algorithm is the same as the optimal solution obtained by the initialized genetic algorithm. 


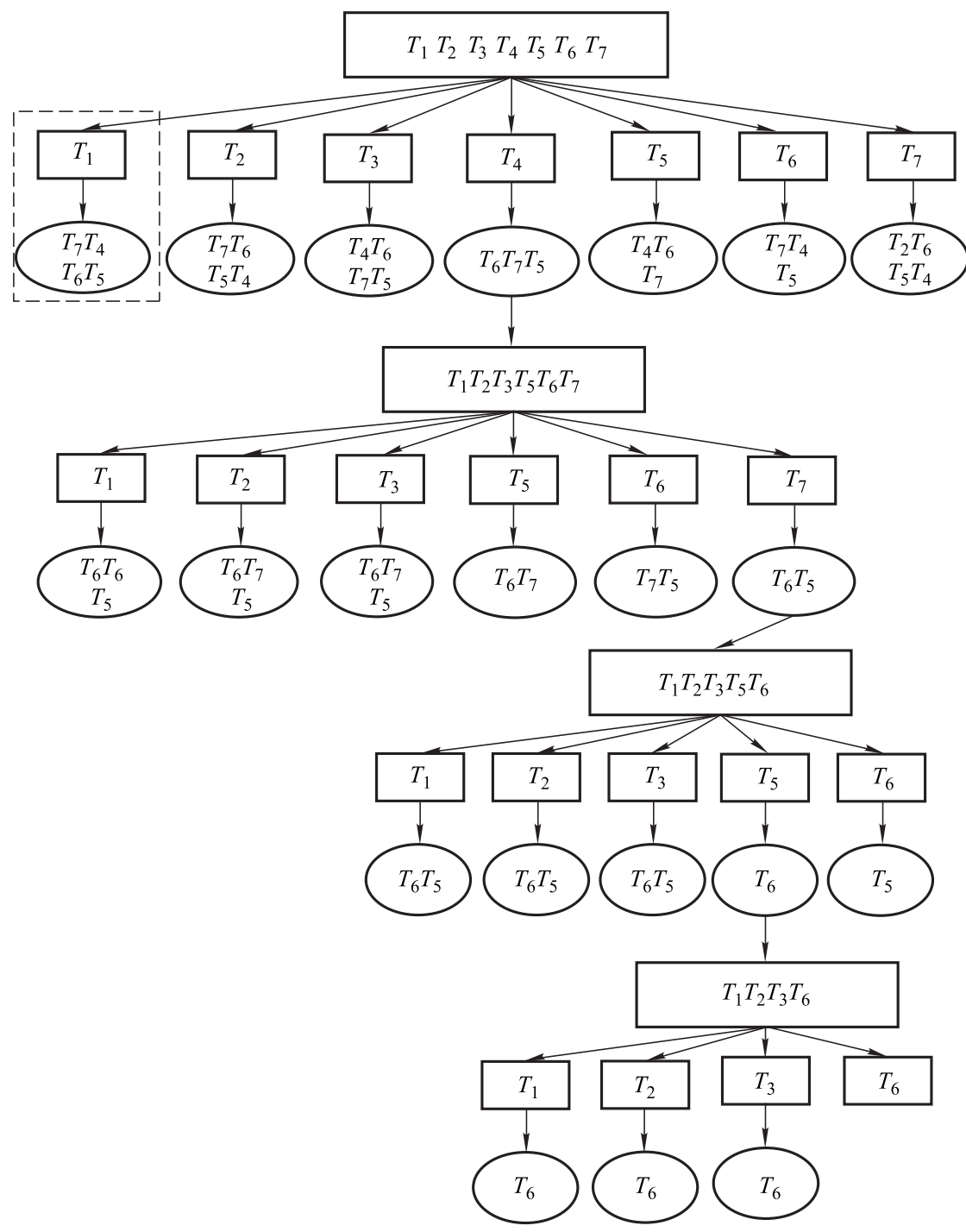

Fig. 1 Optimal test selection process with isolation to faults

\subsection{Optimal test selection with isolation to replaceable unit}

With isolation to replaceable units, the fault pair-test matrices of the two operating modes are obtained by coding the fault-test correlation matrices of operating mode 1 and mode 2 in Table 2, according to (6) and (8), in which the faults that belong to the same unit do not need to be distinguished. In Table 2 , the faults $\left(f_{2}, f_{3}, f_{4}\right),\left(f_{5}, f_{6}, f_{7}\right)$, and $\left(f_{8}, f_{9} f_{10}\right)$ belong to the three units $U_{2}, U_{3}$ and $U_{4}$. Therefore, the final fault pair-test correlation matrix is shown in Table 5 by the fault pair coding, in which the number of fault pairs is $C_{11}^{2}-C_{3}^{2}-C_{3}^{2}-C_{3}^{2}=46$.

According to Table 5, the optimal test selection process by using the rollout heuristic search algorithm is shown in Fig. 2. The final optimal test set is $\left\{T_{7} T_{6} T_{5} T_{4}\right\}$. According to (10), the test $T_{1}$ is selected first by using the benchmark heuristic search strategy, and the final optimal test set is
$\left\{T_{1} T_{7} T_{4} T_{6} T_{5}\right\}$ as shown in Fig. 2. The test number and the test cost are higher than those obtained by the rollout heuristic search algorithm.

Table 5 Fault pair-test correlation matrix

\begin{tabular}{cccccccccc}
\hline $\begin{array}{c}\text { Serial } \\
\text { number }\end{array}$ & Fault pair & $T_{1}$ & $T_{2}$ & $T_{3}$ & $T_{4}$ & $T_{5}$ & $T_{6}$ & $T_{7}$ & Probability \\
\hline 1 & $\left(f_{0} f_{1}\right)$ & 0 & 0 & 0 & 0 & 0 & 1 & 0 & 0.02114 \\
2 & $\left(f_{0} f_{2}\right)$ & 0 & 1 & 0 & 1 & 0 & 0 & 0 & 0.03535 \\
$\vdots$ & $\vdots$ & $\vdots$ & $\vdots$ & $\vdots$ & $\vdots$ & $\vdots$ & $\vdots$ & $\vdots$ & $\vdots$ \\
26 & $\left(f_{3} f_{5}\right)$ & 1 & 1 & 1 & 1 & 0 & 0 & 0 & 0.00003 \\
27 & $\left(f_{3} f_{6}\right)$ & 0 & 0 & 0 & 0 & 0 & 0 & 1 & 0.00028 \\
28 & $\left(f_{3} f_{7}\right)$ & 0 & 0 & 0 & 0 & 0 & 1 & 1 & 0.00005 \\
$\vdots$ & $\vdots$ & $\vdots$ & $\vdots$ & $\vdots$ & $\vdots$ & $\vdots$ & $\vdots$ & $\vdots$ & $\vdots$ \\
43 & $\left(f_{6} f_{10}\right)$ & 1 & 0 & 0 & 1 & 1 & 1 & 0 & 0.00114 \\
44 & $\left(f_{7} f_{8}\right)$ & 0 & 0 & 0 & 0 & 1 & 0 & 0 & 0.00003 \\
45 & $\left(f_{7} f_{9}\right)$ & 0 & 0 & 1 & 1 & 0 & 1 & 0 & 0.00023 \\
46 & $\left(f_{7} f_{10}\right)$ & 1 & 0 & 0 & 1 & 1 & 0 & 0 & 0.00019 \\
\hline
\end{tabular}




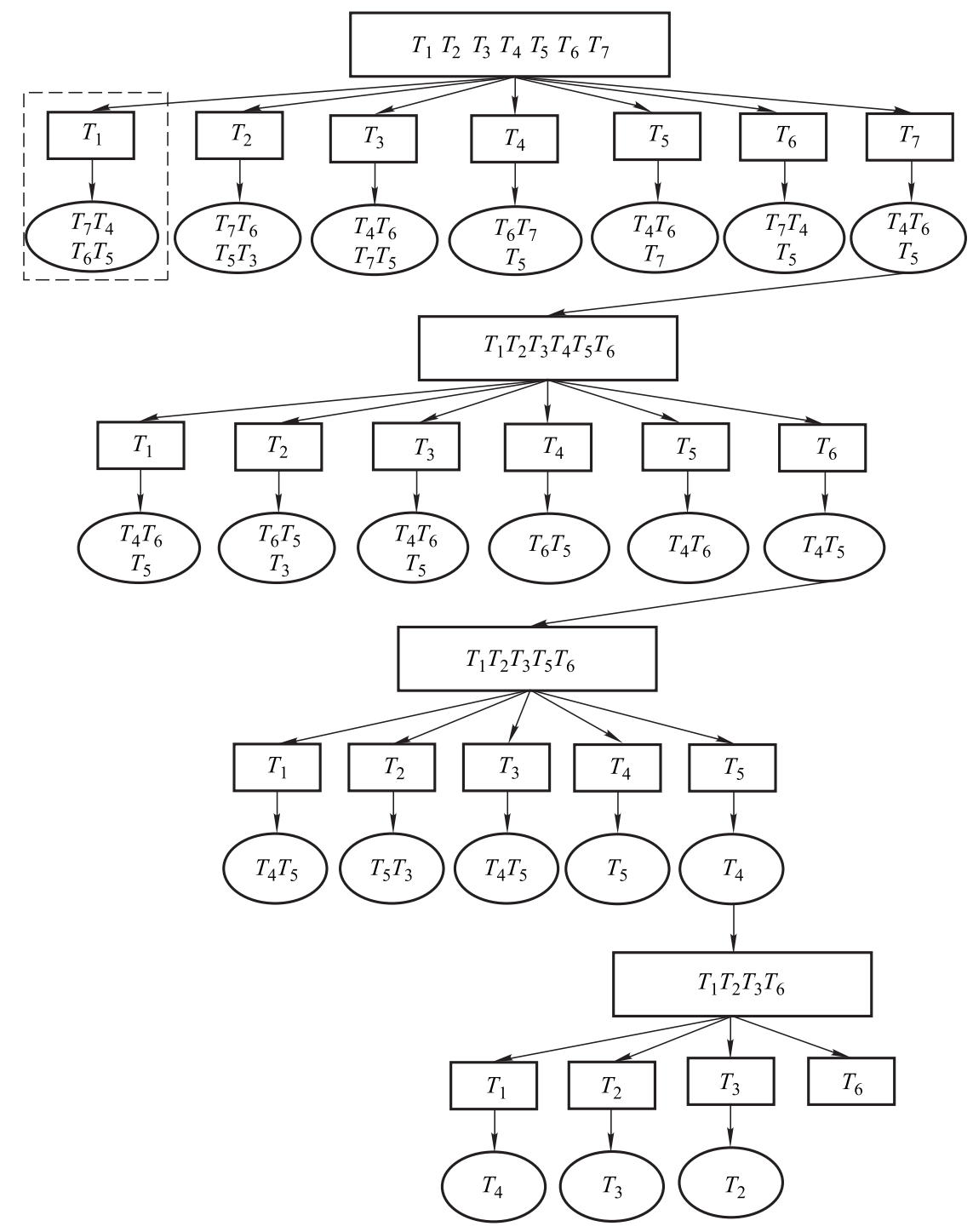

Fig. 2 Optimal test selection process with isolation to replaceable unit

In the same way, the genetic algorithm is used in the test optimization to verify the effectiveness of the proposed algorithm. In the computation process of the genetic algorithm, the parameters in the genetic algorithm are set to the same as those in Section 5.1. The obtained optimal test set isolated from the replaceable unit is $\left\{T_{4} T_{5} T_{6} T_{7}\right\}$. The fault detection rate and the fault isolation rate are both $100 \%$. The optimal solution obtained by the proposed algorithm is the same as the optimal solution obtained by the initialized genetic algorithm.

\section{Practical application}

In order to verify the effectiveness of the proposed algorithm, a certain type of the marine radar system is taken as a practical example for the optimal test selection with faults isolated to the fault mode and the replaceable unit.
When the marine radar system is used to ranging, the display device has two typical operating modes: fixed distance calibration and active distance calibration. The replaceable units, faults and tests in the radar display device are shown in Table 6 . The fault-test correlation matrix in the two operating modes is shown in Table 7; it can be seen that there are a total of 8 replaceable units, 15 failure modes, and 14 initial test sets in the system.

The optimal test set for isolation to fault obtained by the proposed algorithm is $\left\{T_{2} T_{3} T_{4} T_{5} T_{6} T_{7}\right.$ $\left.T_{8} T_{9} T_{10} T_{11} T_{12} T_{13}\right\}$, the test cost is 10.0 , the fault detection rate is $100 \%$, the fault isolation rate is $84.8 \%$, and the fault fuzzy group is $\left\{f_{12} f_{14}\right\}$. The optimal test set isolated to the replaceable unit is $\left\{T_{2} T_{3} T_{4} T_{5} T_{11} T_{12} T_{14}\right\}$, and the fault detection rate and the fault isolation rate are both $100 \%$. 
Table 6 Replaceable units, faults and tests in radar display device

\begin{tabular}{|c|c|c|c|c|c|c|}
\hline Unit name & $\begin{array}{c}\text { Unit } \\
\text { number }\end{array}$ & Fault name & $\begin{array}{c}\text { Fault } \\
\text { number }\end{array}$ & Test name & $\begin{array}{c}\text { Test } \\
\text { number }\end{array}$ & $\begin{array}{l}\text { Test } \\
\text { cost }\end{array}$ \\
\hline Normal system & $U_{0}$ & System normal state & $f_{0}$ & Screen noise detection & $T_{1}$ & 1.0 \\
\hline \multirow{2}{*}{ Bow sign circuit } & \multirow{2}{*}{$U_{1}$} & Bow switch fault & $f_{1}$ & Scan line check & $\overline{T_{2}}$ & 0.5 \\
\hline & & Bow sign circuit fault & $f_{2}$ & Fixed distance check & $T_{3}$ & 0.5 \\
\hline $\begin{array}{c}\text { Video amplifier } \\
\text { circuit }\end{array}$ & $U_{2}$ & Video amplifier circuit fault & $f_{3}$ & Active distance check & $T_{4}$ & 0.5 \\
\hline \multirow{3}{*}{$\begin{array}{l}\text { Fixed distance } \\
\text { calibration circuit }\end{array}$} & \multirow{3}{*}{$U_{3}$} & $\begin{array}{l}\text { Fixed distance calibration } \\
\text { forming circuit fault }\end{array}$ & $f_{4}$ & Bow switch detection & $T_{5}$ & 0.5 \\
\hline & & $\begin{array}{l}\text { Fixed distance controlling } \\
\text { potentiometer fault }\end{array}$ & $f_{5}$ & Bow switch detection & $T_{6}$ & 1.0 \\
\hline & & $\begin{array}{l}\text { Fixed distance standard } \\
\text { linear potentiometer fault }\end{array}$ & $f_{6}$ & $\begin{array}{l}\text { Fixed distance calibration controlling } \\
\text { potentiometer detection }\end{array}$ & $T_{7}$ & 1.0 \\
\hline \multirow{3}{*}{$\begin{array}{l}\text { Active distance } \\
\text { calibration circuit }\end{array}$} & \multirow{3}{*}{$U_{4}$} & $\begin{array}{l}\text { Active distance calibration } \\
\text { forming circuit fault }\end{array}$ & $f_{7}$ & $\begin{array}{l}\text { Fixed distance linear } \\
\text { potentiometer detection }\end{array}$ & $T_{8}$ & 1.0 \\
\hline & & $\begin{array}{l}\text { Active distance controlling } \\
\text { potentiometer fault }\end{array}$ & $f_{8}$ & $\begin{array}{l}\text { Active distance control } \\
\text { potentiometer detection }\end{array}$ & $T_{9}$ & 1.0 \\
\hline & & $\begin{array}{l}\text { Active distance calibration } \\
\text { linear potentiometer fault }\end{array}$ & $f_{9}$ & $\begin{array}{l}\text { Active distance linear } \\
\text { potentiometer detection }\end{array}$ & $T_{10}$ & 1.0 \\
\hline $\begin{array}{l}\text { Synchronous } \\
\text { receiver circuit }\end{array}$ & $U_{5}$ & Synchronous receiver circuit fault & $f_{10}$ & Synchronous receiver circuit detection & $T_{11}$ & 1.0 \\
\hline \multirow{2}{*}{ Scanning circuit } & \multirow{2}{*}{$U_{6}$} & Scanning circuit fault & $f_{11}$ & Scanning circuit detection & $T_{12}$ & 1.0 \\
\hline & & Kinescope damage & $f_{12}$ & Kinescope voltage detection & $T_{13}$ & 1.0 \\
\hline Kinescope & $U_{7}$ & $\begin{array}{l}\text { Kinescope voltage fault } \\
\text { Deflection yoke fault }\end{array}$ & $\begin{array}{l}f_{13} \\
f_{14}\end{array}$ & Square wave detection & $T_{14}$ & 0.5 \\
\hline $\begin{array}{l}\text { Square wave } \\
\text { generator }\end{array}$ & $U_{8}$ & Square wave generator fault & $f_{15}$ & & & \\
\hline
\end{tabular}

Table 7 Fault-test correlation matrix for radar display device

\begin{tabular}{|c|c|c|c|c|c|c|c|c|c|c|c|c|c|c|c|c|c|c|c|c|c|c|c|c|c|c|c|c|c|}
\hline \multirow{2}{*}{ Fault } & \multicolumn{14}{|c|}{ Display device operating under fixed distance calibration } & \multicolumn{14}{|c|}{ Display device operating under active distance calibration } & \multirow{2}{*}{ Probability } \\
\hline & & & & & & & & & & 11 & & & & $\overline{t_{114}}$ & & & & & & 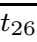 & $t_{0}$ & $t_{0}$ & $t$ & $t_{210}$ & $t_{211}$ & $t_{212}$ & $t_{213}$ & $t_{214}$ & \\
\hline$f_{0} \quad U_{0}$ & 0 & 0 & 0 & 0 & 0 & 0 & 0 & 0 & 0 & 0 & 0 & 0 & 0 & 0 & 0 & 0 & 0 & 0 & 0 & 0 & 0 & 0 & 0 & 0 & 0 & 0 & 0 & 0 & 0.8 \\
\hline$f_{1}$ & 0 & 0 & 0 & 0 & 1 & 1 & 0 & 0 & 0 & 0 & 0 & 0 & 0 & 0 & 0 & 0 & 0 & 0 & 1 & 1 & 0 & 0 & 0 & 0 & 0 & 0 & 0 & 0 & 0.0183 \\
\hline$f_{2}$ & 0 & 0 & 0 & 0 & 1 & 0 & 0 & 0 & 0 & 0 & 0 & 0 & 0 & 0 & 0 & 0 & 0 & 0 & 1 & 0 & 0 & 0 & 0 & 0 & 0 & 0 & 0 & 0 & 0.0073 \\
\hline$f_{3} \quad U_{2}$ & 1 & 0 & 1 & 0 & 1 & 0 & 0 & 0 & 0 & 0 & 0 & 0 & 0 & 0 & 1 & 0 & 0 & 1 & 1 & 0 & 0 & 0 & 0 & 0 & 0 & 0 & 0 & 0 & 0.0045 \\
\hline$\overline{f_{4}}$ & 0 & 0 & 1 & 0 & 0 & 0 & 0 & 0 & 0 & 0 & 0 & 0 & 0 & 0 & 0 & 0 & 0 & 0 & 0 & 0 & 0 & 0 & 0 & 0 & 0 & 0 & 0 & 0 & 0.0224 \\
\hline$f_{6}$ & 0 & 0 & 1 & 0 & 0 & 0 & 0 & 1 & 0 & 0 & 0 & 0 & 0 & 0 & 0 & 0 & 0 & 0 & 0 & 0 & 0 & 0 & 0 & 0 & 0 & 0 & 0 & 0 & 0.0097 \\
\hline$f_{7}$ & 0 & 0 & 0 & 0 & 0 & 0 & 0 & 0 & 0 & 0 & 0 & 0 & 0 & 0 & 0 & 0 & 0 & 1 & 0 & 0 & 0 & 0 & 0 & 0 & 0 & 0 & 0 & 0 & 0.0144 \\
\hline$U_{4}$ & 0 & 0 & 0 & 0 & 0 & 0 & 0 & 0 & 0 & 0 & 0 & 0 & 0 & 0 & 0 & 0 & 0 & 1 & 0 & 0 & 0 & 0 & 1 & 0 & 0 & 0 & 0 & 0 & 0.0035 \\
\hline$f_{9}$ & 0 & 0 & 0 & 0 & 0 & 0 & 0 & 0 & 0 & 0 & 0 & 0 & 0 & 0 & 0 & 0 & 0 & 1 & 0 & 0 & 0 & 0 & 0 & 1 & 0 & 0 & 0 & 0 & 0.0225 \\
\hline$f_{10} \quad U_{5}$ & 0 & 1 & 0 & 0 & 0 & 0 & 0 & 0 & 0 & 0 & 1 & 0 & 0 & 0 & 0 & 1 & 0 & 0 & 0 & 0 & 0 & 0 & 0 & 0 & 1 & 0 & 0 & 0 & 0.0128 \\
\hline$f_{11} \quad U_{6}$ & 0 & 1 & 0 & 0 & 0 & 0 & 0 & 0 & 0 & 0 & 0 & 1 & 0 & 0 & 0 & 1 & 0 & 0 & 0 & 0 & 0 & 0 & 0 & 0 & 0 & 1 & 0 & 0 & 0.0161 \\
\hline$f_{13} \quad U_{7}$ & 0 & 1 & 0 & 0 & 0 & 0 & 0 & 0 & 0 & 0 & 0 & 0 & 1 & 0 & 0 & 1 & 0 & 0 & 0 & 0 & 0 & 0 & 0 & 0 & 0 & 0 & 1 & 0 & 0.0111 \\
\hline$f_{14}$ & 0 & 1 & 0 & 0 & 0 & 0 & 0 & 0 & 0 & 0 & 0 & 0 & 0 & 0 & 0 & 1 & 0 & 0 & 0 & 0 & 0 & 0 & 0 & 0 & 0 & 0 & 0 & 0 & 0.0215 \\
\hline$f_{15} \quad U_{8}$ & 0 & 1 & 0 & 0 & 0 & 0 & 0 & 0 & 0 & 0 & 0 & 0 & 0 & 1 & 0 & 1 & 0 & 0 & 0 & 0 & 0 & 0 & 1 & 1 & 0 & 0 & 0 & 1 & 0.0127 \\
\hline
\end{tabular}

The correlation between the evolution generation and the optimal solution in the evolution process by the genetic algorithm in which the parameters are set to the same as those in Section 5.1 is shown in Fig. 3. The obtained optimization test set isolated from faults is $\left\{T_{3} T_{4} T_{6} T_{7} T_{8}\right.$ $\left.T_{9} T_{10} T_{12}\right\}$, the fault detection rate is $79.85 \%$, the fault isolation rate is $80.96 \%$, and the maximum value of the fitness function is 0.4575 . The obtained optimal test set isolated from the replaceable unit is $\left\{T_{2} T_{3} T_{4} T_{5} T_{11} T_{12} T_{14}\right\}$ by the genetic algorithm in which the parameters are set to the same as those in Section 5.1, the fault detection rate and the fault isolation rate are both $100 \%$. Obviously, the local optimal test set isolated to faults is obtained by the genetic algorithm. If you want to obtain the globally optimal solution, you may try to improve the genetic algorithm by adjusting the crossover, the mutation process, and the fitness 
function, but it is difficult to get the universally adapted parameters of the genetic algorithm and the optimal solution for different fault-test matrices.

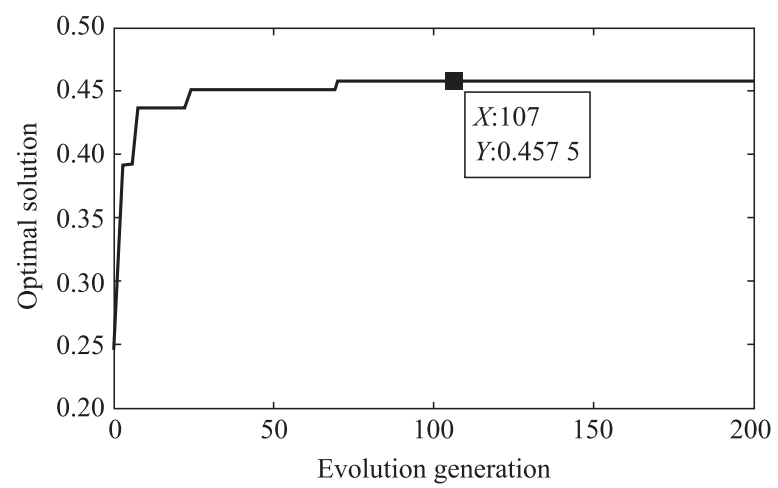

Fig. 3 Correlation between evolution generation and optimal solution in the evolution process

The proposed algorithm is essentially a heuristic search algorithm, and the optimal solution is obtained by seeking a heuristic rule that can generate feasible solutions. By the comparison between the proposed algorithm and the genetic algorithm, we find that the genetic algorithm tends to converge to the local optimal solution of isolation from faults in practical application where the fault-test correlation matrix is large, and the proposed algorithm can effectively mine the fault detection and isolation ability of tests, obtains a better solution of isolation to faults in practical application. The proposed algorithm solves the optimal test selection problems with a higher practicability.

\section{Conclusions}

An optimal test selection method using the fault pair coding and rollout heuristic search algorithm is presented, which combines the factors such as test cost, fault probability, detection and isolation ability of test. Then the effectiveness of the proposed method is verified by the numerical case analysis and practical application. The main conclusions are as follows.

(i) The optimal test selection process including the fault detection and isolation of the multi-operating mode system is transformed into seeking the optimal test set for detecting fault pairs by using the fault pair coding based on the fault-test correlation matrices of the multi-operating mode system.

(ii) The optimal test selection process is a combinatorial optimization. Based on the fault pair-test correlation matrix, the algorithm obtains an optimal test set by searching optimal tests by using the heuristic search algorithm step by step, and using the benchmark optimal search strategy is often difficult to get the optimal test set, thus it is necessary to perform the iterative search. (iii) In the process of the optimal test selection, the optimal test set obtained by the benchmark heuristic search strategy may be selected to the same. That is, in the iterative search, the values of (13) may be the same. Therefore, it needs to choose the best candidate according to (10) to select the best test from excellent candidates.

(iv) When constructing the fault pair-test correlation matrix by using the fault pair coding method, the fuzzy group can be combined that faults with the same rows in the fault pair-test correlation matrix cannot be distinguished to reduce the calculation complexity of coding by reducing the fault pair-test correlation matrix dimension.

\section{References}

[1] TAN X D. Research on key technologies of design for testability for health state evaluation. Changsha, China: National University of Defense Science and Technology, 2013. (in Chinese)

[2] CHENG L Y, LAI X Q. Rapidly transient response with the flexible mode and high-efficiency buck-boost converter. Journal of Xidian University, 2016, 43(5): 93 - 98. (in Chinese)

[3] XIONG X L. On bifurcation of multi operating-mode standalone renewable power generation systems. Nanjing, China: Nanjing University of Aeronautics and Astronautics, 2015. (in Chinese)

[4] WANG B, XU J, WAI R J, et al. Adaptive sliding-mode with hysteresis control strategy for simple multi-mode hybrid energy storage system in electric vehicles. IEEE Trans. on Industrial Electronics, 2017, 64(2): $3592-3600$.

[5] BA T, GAO Y H, ZENG X H, et al. Mode-switch control scheme for hybrid electric vehicle. Journal of Jilin University (Engineering and Technology Edition), 2016, 46(1): 20-26. (in Chinese)

[6] ZHU J, GE Z, SONG Z. Distributed Gaussian mixture model for monitoring plant-wide processes with multiple operating modes. IFAC Journal of Systems and Control, 2018, 6(30): 115.

[7] HILLER B, SAITENMACHER R, WALTHER T. Analysis of operating modes of complex compressor stations. Operations Research Proceedings, 2017: $251-257$.

[8] ZHANG Y, TOMSOVIC K, DJOUADI S M, et al. Hybrid controller for wind turbine generators to ensure adequate frequency response in power networks. IEEE Journal on Emerging and Selected Topics in Circuits and Systems, 2017, 7(3): $1-11$.

[9] PATTIPATI K R, RAGHAVAN V, SHAKERI M, et al. Teams: testability engineering and maintenance system. Proc. of the American Control Conference, 1994: 1989-1995.

[10] SHAKERI M. Advances in system fault modeling and diagnosis. University of Connecticut, 1996.

[11] HASTE D, GHOSHAL S, PATTIPATI K, et al. Flexible integrated system health management for sustainable habitats. Proc. of the AIAA Information Systems, 2018: 1-16.

[12] AASENG G, FRANK J, IATAURO M, et al. Development and testing of a vehicle management system for autonomous spacecraft habitat operations. 2018 AIAA Space and Astronautics Forum and Exposition, 2018: 1-18.

[13] GOULD E. Modeling it both ways: hybrid diagnostic modeling and its application to hierarchical system designs. Proc. of the Auto-Test Conference, 2004: 576-582.

[14] TANG X, XU A, NIU S. KKCV-GA-based method for opti- 
mal analog test point selection. IEEE Trans. on Instrumentation and Measurement, 2017, 66(1): 24-32.

[15] YANG C. Parallel-series multi-objective genetic algorithm for optimal tests selection with multiple constraints. IEEE Trans. on Instrumentation and Measurement, 2018, 67(8): 18591876.

[16] ZHANG S G, PATTIPATI K R, HU Z, et al. Optimal selection of imperfect tests for fault detection and isolation. IEEE Trans. on Systems Man and Cybernetics: Systems, 2013, 43(6): $1370-1384$.

[17] LIU G, LI F, DI P. Research on optimal test selection based on fused algorithm. Computer Science, 2013, 40(6): 54-57. (in Chinese)

[18] DAI X C, NAN J G, HUANG L, et al. An optimal test selection based on improved genetic simulated annealing algorithm. Journal of Air Force Engineering University (Natural Science Edition), 2016, 17(2): 70-75. (in Chinese)

[19] LV X F, ZHOU D Y, TANG Y C, et al. An improved test selection optimization model based on fault ambiguity group isolation and chaotic discrete PSO. Complexity, 2018: 1-10.

[20] DENG S, JING B, ZHOU H. Heuristic particle swarm optimization approach for test point selection with imperfect test. Journal of Intelligent Manufacturing, 2017, 28(1): 37-55.

[21] MA L, LI H J, WANG C G, et al. Optimized test selection method considering the cost of alternative maintenance. Chinese Journal of Scientific Instrument, 2015, 36(2): 280-286. (in Chinese)

[22] HUA J, QIN K Y. Optimal test selection based on improved quantum-inspired evolutionary algorithm. Chinese Journal of Scientific Instrument, 2013, 34(4): 838-845. (in Chinese)

[23] WANG H Q, WANG G W, GUO L H, et al. Optimization selection of test points on complex equipment for discrete firefly algorithm. Optics and Precision Engineering, 2017, 25(5): 1357 - 1367. (in Chinese)

[24] SRIVATSAVA P R, MALLIKARJUN B, YANG X S, et al. Optimal test sequence generation using firefly algorithm. Swarm and Evolutionary Computation, 2013, 8: 44-53.

[25] LIU J M, LIU Y H, FENG F Z, et al. The optimization selec- tion of tests based on greedy algorithm. Acta Armamentarii, 2014, 35(12): 2109-2115. (in Chinese)

[26] LI B L, YANG S M, GUO C. Research on system level equipment test optimization selection based on PHM demand. Measurement \& Control Technology, 2015, 34(11): 144-147. (in Chinese)

[27] GJB 2547A-2012. General requirements for equipment testability. China General Armament Department, China, 2012. (in Chinese)

[28] BERTSEKAS D P, TSITSIKLIS J N, WU C. Rollout algorithms for combinatorial optimization. Journal of Heuristics, 1997, 3(3): $245-262$.

[29] BERTSEKAS D P, CASTANON D A. Rollout algorithms for stochastic scheduling problems. Journal of Heuristics, 1998, 5(5): $89-108$.

[30] GOODSON J C, THOMAS B W, OHLMAN J W. A rollout algorithm framework for heuristic solutions to finite-horizon stochastic dynamic programs. European Journal of Operational Research, 2016, 258(1): 216-229.

[31] MIL-STD-2165A. Military standard testability program for systems and equipments. U.S. Department of Defense, USA, 1995.

\section{Biographies}

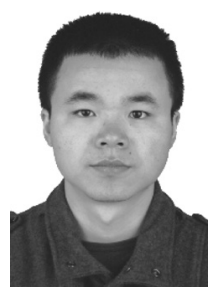

LIU Yuanhong was born in 1987. He received his B.S. degree in vehicle engineering from Military Transportation University, Tianjin, China, in 2009, his M.S. degree in carrier utilization engineering from Military Transportation University, Tianjin, China, in 2011, and his Ph.D. degree in mechanical engineering from Academy of Armored Force Engineering, Beijing, China, in 2015. Since 2016, he is a lecturer in Equipment Engineering College, Chinese People's Armed Police Force Engineering University. His research interests include testability design and fault diagnosis of complex system.

E-mail: qingyun16211@163.com 